\title{
Experience of diagnostics and treatment of infants with ovarian masses
}

\begin{abstract}
Adenomyosis is one of the manifestations of endometriosis that affects the uterus and is characterized by the formation of benign tumors. While the specifics of surgical management of this condition are being continuously debated, the gold standard of surgery is the excision of the affected myometrium. In this study, we discuss the main approaches to adenomyomectomy, and the impact of surgery on female fertility. We also describe a proprietary technique for uterine stitches after surgery.
\end{abstract}

Volume 5 Issue I - 2019

\author{
AN Taits, DO Ivanov, VA Reznik, NN \\ Rukhliada, AA Malysheva, MS Bakhmatskaya, \\ TD Smurova \\ Federal State Budgetary Educational Institution of Higher \\ Education, Saint Petersburg State Pediatric Medical University,
} Russia

Correspondence: NN Rukhliada, Federal State Budgetary Educational Institution of Higher Education, Saint Petersburg Pediatric Medical University of the Ministry of Health of Russia, Russia,Email nickolasr@mail.ru

Received: December 13, 2018 | Published: January 04, 2019

\section{Introduction}

The formation of the ovarian reserve, which is responsible for the fertility of the future woman, occurs during the fetal life. The major ovarian structures are founded in utero and the conditions, under which embryogenesis was proceeding, determine the reproductive potential. ${ }^{1-3}$ The improvement of methods for antenatal imaging of the fetus has led to an increase in the frequency of in utero diagnosis of intra-abdominal cystic masses. The most often, these tumor-like masses in girls have an ovarian origin. ${ }^{1-7}$ Intra-abdominal cystic masses are most often revealed in fetuses during the III trimester of gestational age., ${ }^{3,6,7}$ This abnormality occurs in about one of 2,0002,500 live-born girls. ${ }^{3,6}$

For diagnosing abdominal masses, the ultrasonic method (US), X-ray examination, computed tomography (CT) and magnetic resonance imaging (MRI) are used. The specificity of the fetus and the neonatal period requires minimizing the harmful effects of $\mathrm{x}$-rays, action of drug induced sleep necessary for carrying out the visualization at an early age, as well as the total duration of the diagnostic process when using MRI. Thus, an US examination is optimal for diagnosing ovarian masses in this category of patients. ${ }^{8-20}$ On detection of cystic masses in the abdominal cavity, a differential diagnosis should be carried out to exclude renal malformations, mesenteric cysts, parts of intra-abdominal coccygeal teratomata, lymphangiomata, neuroblastomata. ${ }^{7,15,16}$

Currently, there are US criteria for the evaluation of ovarian cysts: they are divided into simple and complex ones. The latter represent cysts with hemorrhages, suspension, fluid level, septa, calcifications and are predominantly indicative of torsion of the pedicle of an ovarian cyst. It is also necessary to evaluate the cyst pedicle length, which is a predictor of possible torsion and impaired blood flow in the adnexa. Torsion of the pedicle of an ovarian cyst most often occurs in utero. In the neonatal period, this complication develops in very rare cases. ${ }^{10,12,13}$ Despite the rare cases of torsion of the pedicle of a cyst in the fetus during labor, the presence of a mass in the abdominal cavity is not an indication for operative delivery. ${ }^{4}$ An operative delivery should be only considered in case of giant masses that could impede a natural childbirth.
When morphologically assessing ovarian masses in newborns, simple follicular ovarian cysts predominate; to a lesser extent, luteal cysts, benign tumors (serous cystadenomata) and a small percentage of malignant ovarian tumors are revealed. Also, isolated occurrences of endometriomata have been described. . $^{1,2,7,21}$ An ovarian cyst is usually asymptomatic and casually detected during an abdominal ultrasound. In the early neonatal period, this abnormality can be associated with a vivid manifestation of the hormonal crisis in newborns: breast engorgement and vaginal bleeding (Figure 1)., ${ }^{4,11,22-25}$ Despite an improvement in prenatal ultrasonographic diagnosis, the management of fetal ovarian cysts remains controversial. In 2005, based on the Medline database of 420 cases of ovarian cysts, a metanalysis was performed. ${ }^{1}$ In 209 newborns, the cysts regressed spontaneously and in 145 cases, the cysts were complicated by torsion and intracystic hemorrhage. Surgical treatment was performed in 174 newborns. The cysts $<50 \mathrm{~mm}$ regressed spontaneously in $98 \%$ and the cysts $>50 \mathrm{~mm}$ resulted in complications in $93 \%$. After the diagnosis of fetal ovarian cysts $<50 \mathrm{~mm}$, the great majority of authors recommended serial ultrasonic monitoring. The spontaneous regression of a cyst $>50 \mathrm{~mm}$ after aspiration of the cyst content was observed in $89 \%$ of infants. The prenatal aspiration of the ovarian cyst content is effective and safe, but, in later twenty years, it is relatively rarely performed. The spontaneous regression of simple cysts less than $50 \mathrm{~mm}$ does not raise questions, but the observation of complex cysts showed that conservative management could not preserve viable ovarian tissues. $^{12}$ On further observation, the presence of complex cysts resulted in tissue atrophy and loss of ovarian function. Conservative management of complex cysts, despite their sizes, has poor outcomes. The visualization of the ovaries on both sides was successful only in $60 \%$ of adolescents. ${ }^{17}$ Torsion of the pedicle of an ovarian cyst may spontaneously regress, but it may cause such severe complications as self-amputation of the adnexa and their migration in the different regions of the abdominal cavity, intestinal obstruction, labial edema on the side of the affected ovary, respiratory disorders and provoke sudden infant death (Figure 2). ${ }^{11,12,15,16}$ Surgical treatment is performed laparoscopically; giant masses may be transcutaneously prepunctured. All the surgical interventions are performed with maximal ovarian tissue preservation. The surgical team should include an obstetrician-gynecologist. ${ }^{4}$ Histologically, these are most often simple 
follicular cysts with hemorrhages, calcifications and necrosis in case of torsion (Figure 3 ). The aim of the paper was to make a retrospective analysis and to assess the disease course, methods of diagnosis and treatment for girls with detected tumor-like abdominal masses.

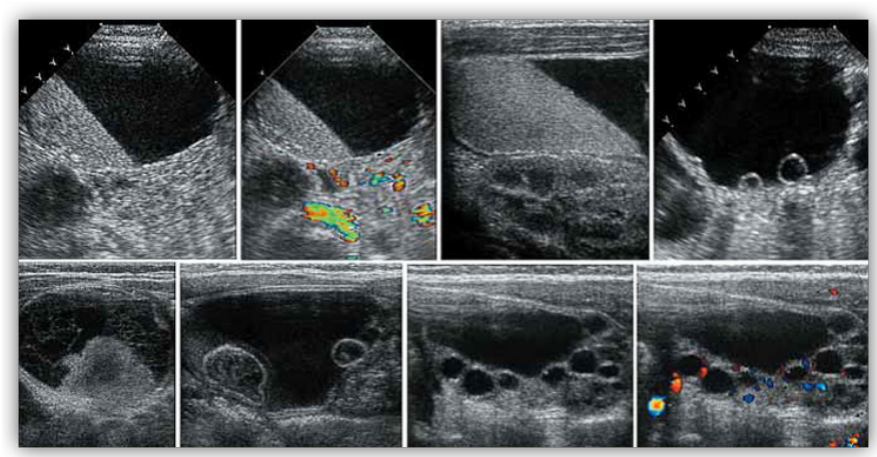

Figure I Different variation of ovarian cysts in newborns.

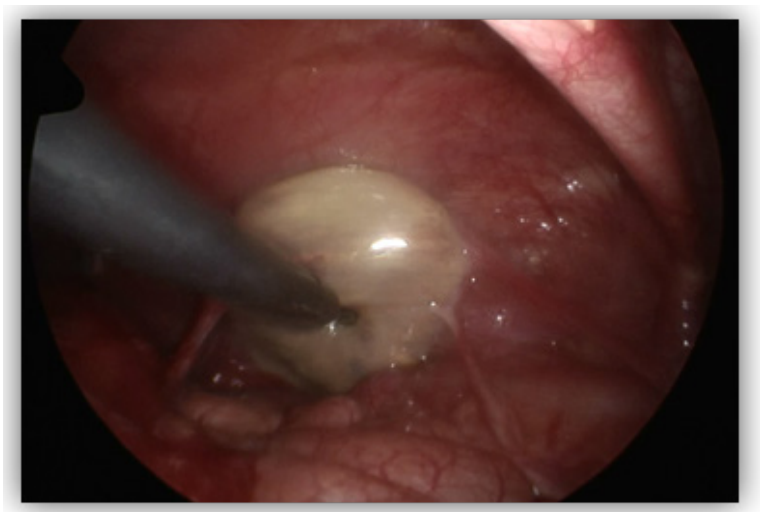

Figure 2 Torsion of the pedicle of an ovarian cyst.

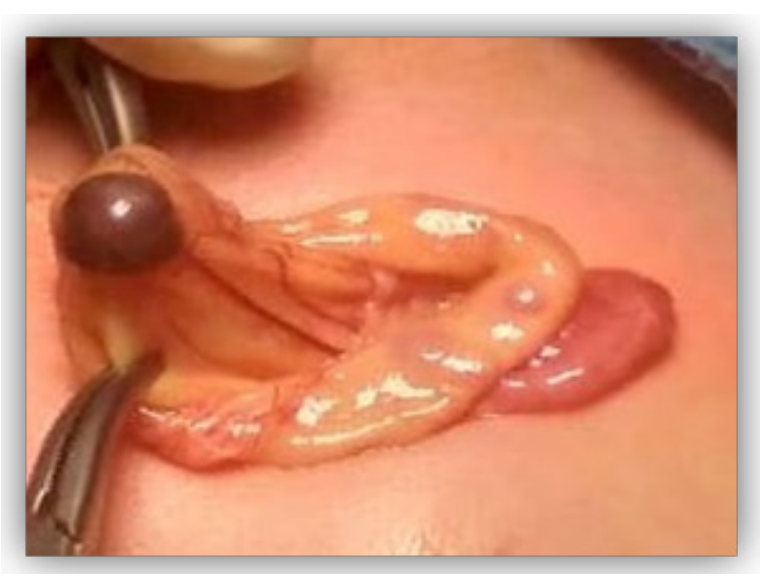

Figure 3 Necrosis of the cyst wall.

\section{Materials and methods}

Nineteen girls were enrolled in our study: Seventeen newborns and 2 female infants ( 1 day to 2 months and 6 days) that received medical care at the Prenatal Center (PC) of the Federal State Budgetary Educational Institution of Higher Education Saint Petersburg State Pediatric Medical University (FSBEI HE SPBSPMU) over the period of 2014-2018. The literature review, analysis and assessment of the disease course, the diagnostic and treatment methods was performed. The examination of the girls included the analysis of pregnancy course, history taking, general clinical tests, pelvic and abdominal ultrasound, abdominal MSCT, histological examination of surgical material. Surgical treatment was provided to 18 infant girls; 1 child was under medical supervision. Only in one case, the intra-abdominal tumor mass had not an ovarian origin. This was an enterocystoma (malformation of the colon). In most cases, follicular cysts were revealed.

\section{Results of the study}

In 17 girls, the abdominal masses were diagnosed in utero in the III trimester of pregnancy during the US examination (gestational age of 30 to 34 weeks) (Figure 4). In 17 women, the pregnancy was spontaneous; in 2 women, it was a result of IVF. Five patients received progesterone preparations due to threatened miscarriage at different gestational ages. The most frequent diseases during pregnancy were STDs (newly diagnosed mycoplasmosis sanitated during pregnancy), obesity.

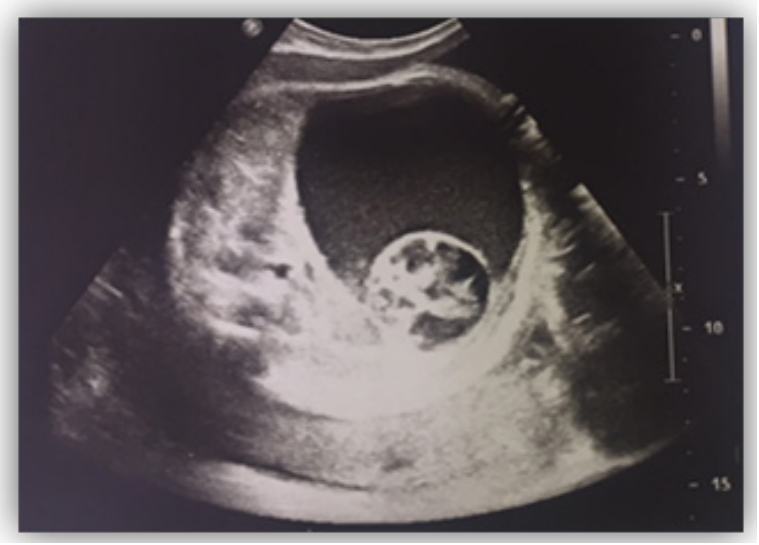

Figure 4 Complex cysts (33 weeks of pregnancy).

In one case, MSCT was performed in utero. During the US examination, 12 girls had complex cysts: there were such signs as parietal inclusions, suspension, septa (Figure 5). In 2 girls, simple cysts were detected - round-shaped, thin-walled, homogeneous masses; the dimensions ranged from 2.5 to $9.5 \mathrm{~cm}$. During dynamic follow-up in the remaining gestational period before and after delivery, in the neonatal period, no cyst changes were revealed. There were no fetal indications for operative delivery; the operative delivery was only performed for obstetric indications. Seventeen newborns were carried to full term; 2 were premature infants born at the gestational age of 28 and 34 weeks. All the full-term girls were born in a satisfactory condition; in the preterm children, the severity of their condition was due to the prematurity. In all cases, the disease course was asymptomatic. Unilateral masses were revealed in 17 cases, bilateral ones - in 2 cases. In 5 newborns, there were remarkable manifestations of the hormonal crisis in the newborns, such as: breast engorgement, bloody vaginal discharge, abundant mucous discharge from the genital tract.

Surgical treatment was performed in 18 patients. Surgical treatment was performed using the hybrid method via the umbilical incision. This technique is minimally invasive and has all the advantages of laparoscopy. Eleven girls underwent tubovarioectomy; there were 7 ovarian cystectomies and 1 removal of the enteric cyst. In a large percentage of cases, preserving the adnexa seemed to be impossible (Figures 6 \& 7). 


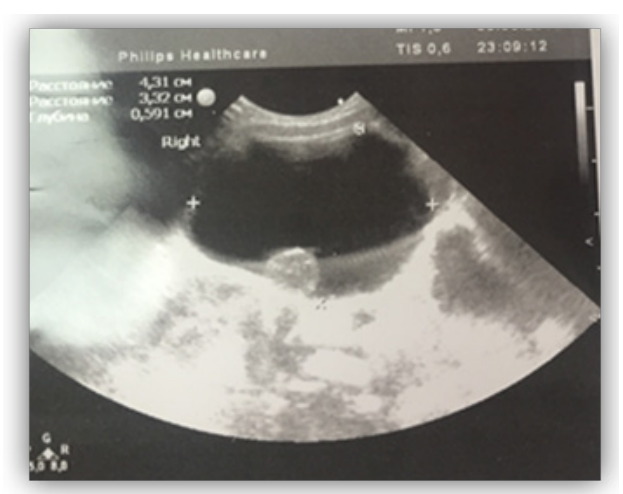

Figure 5 Complex cysts the newborn period.

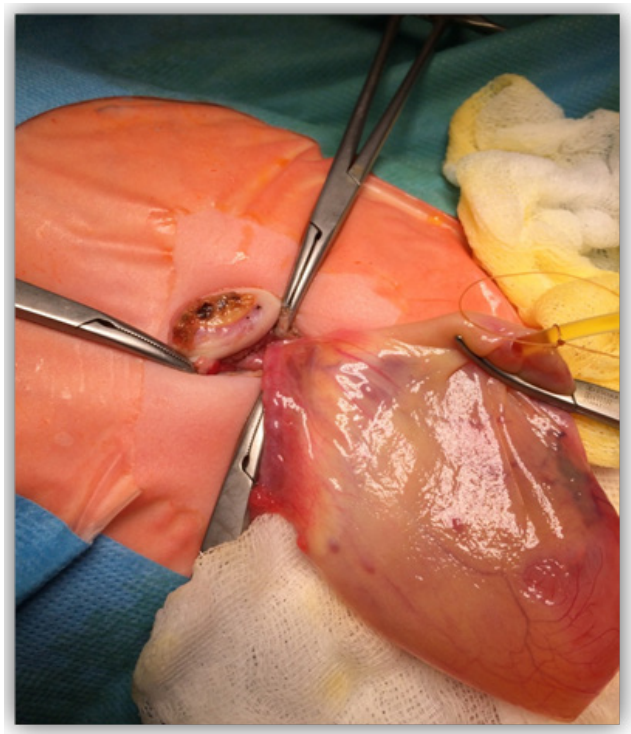

Figure 6 Hybrid method via the umbilical incision. Cystectomy

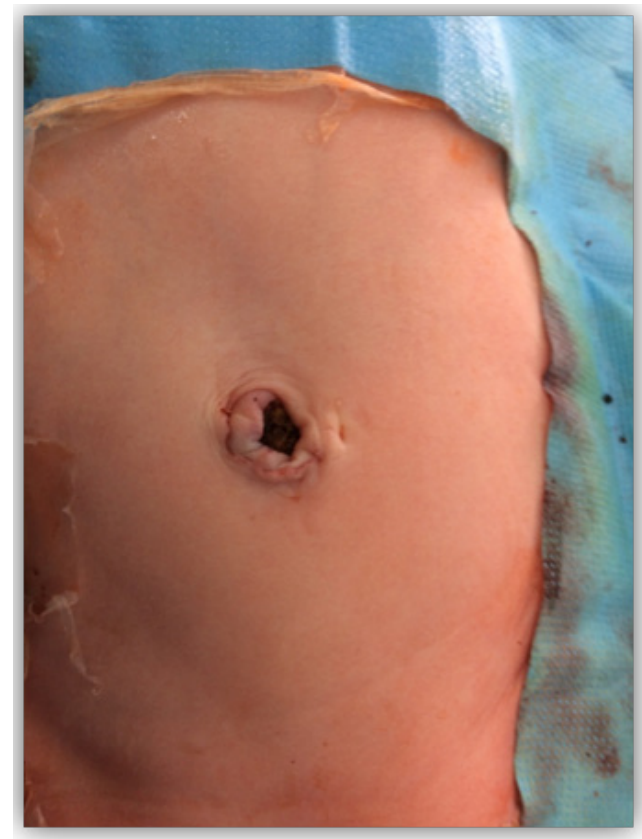

Figure 7 View of operational access after surgery.

\section{Conclusion}

Histologically follicular cyst in 10 children (8-with parietal necrosis, 2-without necrosis), serous cyst in 4 girls; in 1 child, the endometrioid cyst was confirmed; colonic cyst -1 . The combination of the cystic lesion of both ovaries was observed in 1 girl (in one ovary, there was a follicular cyst; in the other, a mixed tumor consisting of germ cells and stomal cells of the genital ridge). The girls with ovarian tumor-like masses were put under dispensary surveillance by a gynecologist.

\section{Acknowledgments}

\section{None}

\section{Conflicts of interest}

The author declares there are no conflicts of interest.

\section{References}

1. Słodki M, Respondek-Liberska M. Fetal ovarian cysts--420 cases from literature--metaanalysis 1984-2005. Ginecol Pol. 2012;78(4):324-328.

2. Adamyan LB, Bogdanova EA, Korotkova SA, et al. Congenital ovarian cysts in infants. Probl reproductions. 2006;6:111-115.

3. Muslimova S Yu, Latypova GG, Uvarova EV. Features of diagnosis and treatment of tumors and tumor-like formations of the ovaries in newborns and infants. Reproductive health of children and adolescents. J Minim Invasive Gynecol. 2017;24(5):859-862.

4. Ladenhauf HN, Brandtner MG, Ardelean MA, et al. Laparoscopic Management of Autoamputated Ovary in Newborns: A Report of 2 Cases. J Pediatr Adolesc Gynecol. 2017;30(4):449-455.

5. Aydin BK, Saka N, Bas F, et al. Evaluation and Treatment Results of Ovarian Cysts in Childhood and Adolescence: A Multicenter, Retrospective Study of 100 Patients. J Pediatr Adolesc Gynecol. 2017;30(4):449-455

6. Llorens Salvador R, Sangüesa Nebot C, Pacheco Usmayo A. Neonatal ovarian cysts: ultrasound assessment and differential diagnosis. Radiologia. 2017;59(1):31-39.

7. Kim HS, Yoo SY, Cha MJ. Diagnosis of neonatal ovarian torsion: Emphasis on prenatal and postnatal sonographic findings. $J$ Clin Ultrasound. 2016;44(5):290-297.

8. Cho MJ, Kim DY, Kim SC. Ovarian Cyst Aspiration in the Neonate: Minimally Invasive Surgery. J Pediatr Adolesc Gynecol. 2015;28(5):348353.

9. Ozcan HN, Balci S, Ekinci S, et al. Imaging Findings of Fetal-Neonatal Ovarian Cysts Complicated With Ovarian Torsion and Autoamputation. AJR Am J Roentgenol. 2015;205(1):185-189. 